\title{
The Prognostic Value of Preoperative Serum Lactate Dehydrogenase (LDH) Levels in Patients Underwent Curative-intent Hepatectomy For Colorectal Liver Metastases: A Two-Center Cohort Study
}

\section{Long Bai}

Sun Yat-sen University Cancer Center

Ze-Yu Lin

Sixth Affiliated Hospital of Sun Yat-sen University

Yun-Xin Lu

Sun Yat-sen University Cancer Center

Qin Chen

Sixth Affiliated Hospital of Sun Yat-sen University

Han Zhou

Sixth Affiliated Hospital of Sun Yat-sen University

Qi Meng

Sun Yat-sen University Cancer Center

Chun-Ping Lin

Jieyang Affiliated Hospital, Sun Yat-sen University

Wan-Lan Huang

Jieyang Affiliated Hospital, Sun Yat-sen University

Yun-Le Wan

Sixth Affiliated Hospital of Sun Yat-sen University

Zhi-Zhong Pan

Sun Yat-sen University Cancer Center

De-Shen Wang ( $\sim$ wangdsh@sysucc.org.cn )

Sun Yat-sen University Cancer Center

\section{Research Article}

Keywords: colorectal liver metastases (CRLM), hepatectomy, lactate dehydrogenase (LDH), circulating biomarker

Posted Date: April 29th, 2021 
DOI: https://doi.org/10.21203/rs.3.rs-236654/v2

License: (c) (1) This work is licensed under a Creative Commons Attribution 4.0 International License. Read Full License

Version of Record: A version of this preprint was published at Cancer Medicine on October 12th, 2021. See the published version at https://doi.org/10.1002/cam4.4315. 


\section{Abstract}

Background. The prognostic value of lactate dehydrogenase (LDH) in colorectal cancer patients has remained inconsistent between non-metastatic and metastatic settings. So far, very few studies have included LDH in the prognostic analysis of curative-intent surgery for colorectal liver metastases (CRLM).

Patients and Methods. 580 consecutive metastatic colorectal cancer patients who underwent curativeintent CRLM resection from Blinded for peer review (434 patients) and Blinded for peer review (146 patients) treated in 2000-2019 were enrolled. Overall survival (OS) was the primary endpoint. Cox regression model was performed to identify the prognostic values of preoperative serum LDH levels and other clinicopathology variables. A modification of the established Fong CRS scoring system comprising LDH was developed within this Chinese population.

Results. At the median follow-up time of 60.5 months, and median OS was 59.5 months in the pooled cohort. In the multivariate analysis, preoperative $\mathrm{LDH}>$ upper limit of normal $(250 \mathrm{U} / \mathrm{L})$ was the strongest independent prognostic factor for OS (HR 1.73, 95\% confidence interval [CI], 1.22-2.44; $P<.001$ ). Patients with elevated LDH levels showed impaired OS than patients with normal LDH levels ( 27.6 months vs. 68.8 months). Five-year survival rates were $53.7 \%$ and $22.5 \%$ in the LDH-normal group and LDH-high group, respectively. Similar results were also confirmed in each cohort. In the subgroup analysis, LDH could distinguish the survival regardless of most established prognostic factors (number and size of CRLM, surgical margin, extrahepatic metastases, CEA and CA19-9 levels, etc.). Integrating LDH into the Fong score contributed to an improvement in the predictive value.

Conclusion. Our study implicates serum LDH as a reliable and independent laboratory biomarker to predict the clinical outcome of curative-intent surgery for CRLM. Composite of LDH and Fong score is a potential stratification tool for CRLM resection. Prospective, international studies are needed to validate these results across diverse populations.

\section{Introduction}

Colorectal cancer (CRC) is the third most common cancer and the second leading cause of cancer-related mortality worldwide (1-3). The liver is the primary life-limiting distant metastatic site for CRC (4). About a quarter of CRC patients present concurrent liver metastases, and over half will develop liver metastases through the course of diseases (5) . Surgical excision-based locoregional therapy remains the only possible curative option for colorectal liver metastases (CRLM) (6). However, only about $20 \%$ of CRLM patients are candidates for curatively intended liver resection at diagnosis (7). Whereas a growing number of curative hepatectomy has been achieved through multi-disciplinary therapy within the latest decade, most patients $(50 \%-80 \%)$ would develop further recurrence $(8,9)$. The survival outcomes derived from different studies remain heterogeneous, with 5-year survival rates ranging from $25 \%$ to $60 \%$ (10-12). Thus, a better selection of patients before initiating treatment is needed to refine the therapeutic decisions. 
Recent studies have shown that apart from conventional clinicopathology variables, gene expression signatures, intratumoral immune cell infiltrations, and circulating tumor cells also have a prognostic impact on colorectal cancer $(10,11,13)$. In particular, the serum biochemical markers, namely Gammaglutamyl transferase (GGT), alkaline phosphatase (ALP), and lactate dehydrogenase (LDH), also gained momentum as prognostic indicators for $\operatorname{mCRC}(14,15)$.

As the key enzyme in aerobic and anaerobic glycolysis, LDH plays a pivotal role in tumor metabolism by mediating the conversion of pyruvate and lactate (16). Evidence is emerging that LDH is closely related to hypoxia, angiogenesis, inflammation, and immune status in the tumor microenvironment (TME). High serum LDH levels indicate poor prognosis among various cancer entities, and promote resistance to chemo/radio/targeted therapy (17-19). However, the prognostic value of LDH in CRC has remained inconsistent between non-metastatic and metastatic settings (20-22). Elevated circulating LDH levels were reported to be an adverse prognostic factor in unresectable CRLM patients receiving systemic therapy or hepatic arterial infusion (23-25). In contrast, this effect was not evident for the overall survival of non-metastatic CRC patients $(26,27)$. Moreover, very few studies have included LDH in the prognostic analysis of curative-intent surgery for CRLM. Therefore, it remains to be determined whether preoperative LDH levels could predict the outcome of complete CRLM resection, in which situation patients usually achieve a no-evidence-of-disease (NED) status.

To address this issue, we performed this two-center, retrospective observational study in a cohort of 580 patients with resected CRLM. Our objectives are (a) to evaluate the prognostic impact of preoperative serum LDH levels on curative-intent surgery for CRLM; (b) to integrate LDH into the established Fong scoring system within this Chinese population to improve patient stratification for CRLM resection.

\section{Materials And Methods}

\section{Study population}

This two-center, retrospective cohort study enrolled 580 histologically proven CRLM patients who underwent curative-intent hepatectomy in Blinded for peer review (cohort 1) and Blinded for peer review (cohort 2). Cohort 1 included 434 patients from September 2000 to December 2016, while cohort 2 included 146 patients from August 2012 to June 2019. Detailed clinical information (pre-operation and post-operation clinicopathological data, blood examination, follow-up information, etc.) was retrieved from electronic and paper-based medical records from each center. The preoperative LDH values had to be available to be eligible for inclusion.

The exclusion criteria were listed as follows: 1) Peritoneal metastasis; 2) previous history of hepatectomy; 3) R2 resection of liver metastases; 4) ablation of metastatic sites or transcatheter hepatic arterial chemoembolization (TACE) within four weeks of study entry; 5) patients in inflammatory conditions; 6) previous history of malignant tumor. 
The study was approved by the ethics committee of both centers and complied with the Declaration of Helsinki.

\section{Follow-up}

Overall survival (OS) was defined as the time from hepatic resection to death from any cause or latest follow-up. Recurrence-free survival (RFS) was measured from the date of hepatic resection to confirming recurrence or death from any reason, whichever occurred first. Patients were followed up through outpatient clinical visits or via telephone. The follow-up starts one month after the operation and ends when tumor relapse or death was verified, while subjects who were lost or still alive at the date of the last contact were considered censored.

\section{Blood sample test}

Data from blood examination (blood routine tests, blood chemistry tests, and tumor marker tests) were eligible for analysis if performed within two weeks before hepatectomy. The blood examination was performed by each center's laboratory. Enrolled patients were divided into LDH-normal and LDH-high groups, using the ULN (upper limit of normal) established by each center's laboratory as the cutoff value, in anticipation of elaborating a practical clinical tool for future use. The ULN of LDH at both centers was $250 \mathrm{U} / \mathrm{L}$. Preoperative immune/inflammation-related factors (including neutrophil, lymphocyte, monocyte, and platelet counts, LMR, LNR and LPR, and C-reaction protein) were collected. LMR, LNR and LPR were defined as absolute lymphocyte count divided by absolute monocyte, neutrophil, and platelet count, respectively.

\section{Modified Clinical Risk Score establishment and validation}

The clinical risk score (CRS) was calculated according to the criteria initiated by Yuman Fong (28). Briefly, five clinical criteria: primary lymph node-positive, the disease-free interval from the diagnosis of primary tumor < 12 months, number of CRLM > 1, maximum CRLM diameter $>5 \mathrm{~cm}$, preoperative CEA levels > $200 \mathrm{ng} / \mathrm{ml}$, were assigned one point for each and total scores were defined as CRS.

We integrated preoperative LDH levels into the CRS model to test whether the predictive ability improved. Two models were established: (a) LDH was added to the CRS model (LDH-CRS, LDH > ULN was assigned one point); (b) preoperative CEA levels were replaced by LDH levels (modified CRS [mCRS], LDH > ULN was assigned one point).

The discriminatory ability of models was assessed by area under curve (AUC) in the time-dependent receiver operating characteristic (ROC) analysis. Harrell's discrimination concordance index (C-index, which is defined as the probability that predictions and outcomes are concordant) was employed to validate the predictive ability of the models.

\section{Statistical analysis}


Patients' characteristics between different groups were compared with student's t-test, chi-square, Wilcoxon rank-sum test, or Kruskal-Wallis test as statistically appropriate. The survival curves were generated using the Kaplan-Meier method and compared with the log-rank test in terms of RFS and OS.

OS was the primary endpoint. To identify independent prognostic predictors, univariate and multivariate Cox proportional hazard regression analyses were performed. The associations between baseline clinicopathologic variables (age, gender, primary tumor location, grade of differentiation, pathology, $\mathrm{T}$ and $\mathrm{N}$ stage of primary tumor, preoperative CEA and CA19-9 levels, LDH level, number of CRLM, maximum diameter of CRLM, extrahepatic metastases, surgical margin of CRLM, preoperative chemotherapy, disease-free interval from discovery of primary tumor to liver metastases) and survival outcome were explored and quantified by hazard ratios (HRs) and corresponding $95 \%$ confidence intervals (Cls). Parameters with $P<0.10$ in the univariate analysis were selected and further included in the multivariate analysis, relying on the ENTER algorithm with a selected level of 0.05 . In the multivariable analysis for pooled population, the cohort was obligated to be an adjustment factor to exclude the confounding factor of different affiliates.

KRAS and BRAF mutation was not considered for Cox regression analysis because it was not available for all patients, especially for patients in cohort 2 (Table 1). Hence a sensitivity analysis in cases with available data of KRAS mutation status was performed in a multivariable model.

Furthermore, subgroup analyses were carried out stratified by demographic and clinicopathologic variables, and presented by forest plots.

As for the comparison of time-dependent AUC between different models, the Wilcoxon matched-pair signed-rank test was applied. Time-dependent AUC was calculated by the Package timeROC (version 0.4). The C-index was calculated by the Package rms (version 5.1-3.1). Statistical analyses were conducted with the SPSS software version 19 (SPSS, Chicago, Illinois, USA), STATA (Release 14.2; StataCorp LP, College Station, TX) and GraphPad Prism 7.0.

\section{Results}

\section{Characteristics of patients}

A total of 580 consecutive CRLM patients at two Chinese medical centers were enrolled. 434 patients were from cohort 1 , and 146 patients were from cohort 2 . Clinicopathology and treatment characteristics were listed in Table 1. All enrolled patients were Chinese individuals, the average age at diagnosis was 59. The median follow-up time was 60.5 months, whereas median OS was 59.5 months ( $95 \% \mathrm{Cl}, 58.4-70.6)$ in the pooled cohort, 58.9 months $(95 \% \mathrm{Cl}, 46.2-71.6)$ in cohort 1 and 63.3 months $(95 \% \mathrm{Cl}, 61.3-67.8)$ in cohort 2, respectively (Supplementary Table 1 ).

Overall, clinical features were well balanced between the two cohorts, except that patients in cohort 1 had a higher proportion of synchronous CRLM ( $95.2 \%$ vs. $69.4 \%)$, T4 stage of primary tumor $(83.2 \%$ vs. $64 \%)$, 
well to moderately differentiated pathology ( $87.7 \%$ vs. $76.3 \%)$, and LDH levels above ULN $(23.3 \%$ vs. $13.6 \%$ ) than patients in cohort 2 (Supplementary table 1 ).

\section{LDH levels and correlations with clinical characteristics}

The relationship between serum LDH and clinicopathological parameters was detailed in Table 2. In summary, serum LDH levels showed no statistical difference when stratified by demography characteristics (age, gender), primary tumor characteristics (tumor location, pathology differentiation, $\mathrm{T}$ and $\mathrm{N}$ stage, KRAS and BRAF mutation), and metastatic site characteristics (presence of extrahepatic disease, number of CRLM, perioperative chemotherapy).

However, we observed patients with a maximum diameter of $\mathrm{CRLM} \leq 2.5 \mathrm{~cm}$ (the median diameter) had a higher proportion of elevated LDH levels than patients with a maximum diameter of CRLM > median $(24.2 \%$ vs. $9.7 \%, P<.001)$. Patients with elevated CEA also had a greater possibility of having elevated LDH than those with normal CEA levels $(18.9 \%$ vs. $10.7 \%, P=0.011)$. A similar trend was observed for CA19-9 levels $(P=0.006)$. Patients with synchronous CRLM had a higher proportion of elevated LDH than those with metachronous CRLM $(17.9 \%$ vs. $10.1 \%, P=0.033)$. In addition, patients with CRS of $4-5$ had higher proportion of elevated LDH than patients with CRS $2-3$ or CRS $0-1$ ( $51.2 \%$ vs. $14.2 \%$ vs. $8.7 \%$; $P<$ $.001)$.

\section{Cox regression analysis of relapse-free survival and overall survival}

Due to some missing data for the baseline variables (details in Table 1), 490 patients were finally included in the multivariable model. Elevated preoperative LDH levels (defined as LDH > ULN) was found to be the strongest prognostic factor for OS (Table 3).

In the univariate analysis, age, pathology differentiation, $T$ stage of the primary tumor, lymph node metastases of the primary tumor, preoperative CEA and CA19-9 level, number of CRLM, maximum diameter of CRLM, presence of extrahepatic metastases, preoperative chemotherapy, R0 resection margin, and LDH levels were significant predictors for OS.

After adjusted for the above clinicopathologic parameters, eight factors were ultimately identified as independent prognostic makers for $\mathrm{OS}$ in the multivariate analysis: age $(\mathrm{HR}, 1.03 ; 95 \% \mathrm{Cl}, 1.01-1.04 ; P<$ $.001)$, lymph node metastasis of the primary tumor (HR, 1.70; $95 \% \mathrm{Cl}, 1.27-2.27 ; P<.001)$, preoperative CA19-9 (HR, 1.47; 95\% Cl, 1.09-1.98; $P=0.012)$, the number of CRLM (HR, 1.13; 95\% Cl, 1.07-1.20; $P<$ $.001)$, the maximum diameter of CRLM (HR, 1.07; 95\% Cl, 1.01-1.13; $P<.001)$, extrahepatic disease (HR, $1.61 ; 95 \% \mathrm{Cl}, 1.03-2.54 ; P=0.039), \mathrm{R} 0$ resection margin ( $\mathrm{HR}, 0.56 ; 95 \% \mathrm{Cl}, 0.37-0.84 ; P=0.006)$, and elevated preoperative LDH levels (HR, 1.73; 95\% Cl, 1.22-2.44; $P<.00781)$.

In the stratified analyses for each cohort, LDH remained its independent prognostic value for OS in the multivariate analysis, both in cohort $1(\mathrm{HR}, 1.77 ; 95 \% \mathrm{Cl}, 1.17-2.69 ; P<.001$; Supplementary Table 2$)$ and cohort 2 (HR, 3.71; $95 \% \mathrm{Cl}, 1.75-7.89 ; P=0.001$; Supplementary Table 3) 
In terms of RFS, LDH remained an independent predictor in the multivariate analysis $(\mathrm{HR}, 1.53 ; 95 \% \mathrm{Cl}$, 1.01-2.03; $P=0.042$ ), along with lymph node metastases of primary tumor, number of CRLM, and maximum diameter of CRLM (supplementary Table 4).

Additionally, in the sensitivity analysis in cases with available data of KRAS mutation status, only number and size of CRLM were independent predictors for OS in multivariable models, probably due to the limitation of sample size (Supplementary Table 5).

\section{Survival outcomes according to LDH levels and subgroups analysis}

In the pooled cohort, patients with elevated LDH showed impaired OS compared with patients with normal LDH levels (27.6 months vs. 68.8 months; HR, 2.51, 95\% Cl, 1.88-3.36; $P<.001$ ). Survival rates at 5 years in the LDH-normal and LDH-high group were $53.7 \%$ and $22.5 \%$, respectively. In the stratified analysis, cohort 1 (25.0 months vs. 63.6 months; $\mathrm{HR}, 2.41,95 \% \mathrm{Cl}, 1.72-3.39 ; P<.001)$ and cohort 2 (27.8 months vs. not reached; $\mathrm{HR}, 3.16,95 \% \mathrm{Cl}, 1.75-5.70 ; P<.001)$ demonstrated similar results as the pooled cohort (Fig. 1).

On the other hand, patients with elevated LDH had significantly shorter RFS (8.5 months vs. 22.0 months; $\mathrm{HR}, 2.11,95 \% \mathrm{Cl}, 1.54-2.89 ; P<.001$ ) than patients with normal LDH levels in cohort 1 (Supplementary Fig. 1).

Subgroup analyses revealed that LDH produced consistent prognostic value across patient subgroups stratified by age, sex, primary tumor characteristics (location, $\mathrm{T}$ and $\mathrm{N}$ stage), liver metastases characteristics (number, maximum diameter, surgical margin, disease-free interval from primary tumor, extrahepatic disease), perioperative chemotherapy, preoperative CEA and CA19-9 levels, even by Fong score. The forest plots provided a clear trend that patients with lower LDH levels obtained better survival benefit from hepatectomy for OS (Fig. 2).

\section{Survival outcomes assessed by CRS, LDH-CRS and mCRS}

OS stratified by different risk scores as defined by CRS and LDH-CRS were demonstrated by Kaplan-Meier curves in the pooled cohort. Median OS of the risk scores 0-5 in the CRS model was not reached, not reached, 64.5 months, 41.8 months, 27.6 months, 44.8 months, respectively. Median OS of risk scores 0-6 in the LDH-CRS model were not reached, not reached, 77.6 months, 41.6 months, 41.8 months, 24.2 months, 27.5 months, respectively. While median OS of the risk scores $0-5$ in the mCRS model were not reached, not reached, 77.6 months, 39.5 months, 24.2 months, 27.5 months, respectively.

Particularly, LDH-CRS and mCRS identified a relatively higher proportion of patients in the high-risk group (score of 4-6) than CRS (13.2\% [67/506] vs. $12.0 \%$ [63/526] vs. 8.5\% [43/506]). Moreover, the median OS of patients in the high-risk group was numerically shorter in mCRS than in LDH-CRS and CRS (21.8 months vs. 27.8 months vs. 27.8 months) (Fig. 3). 
Receiver operating characteristic (ROC) analysis for the comparison of CRS and LDH-CRS in prediction ability

Time-dependent ROC analysis displayed that LDH-CRS and mCRS exhibited a better predictive value than CRS in the pooled cohort for OS $(P=0.016)$. In the CRS model, the C-index of the 5 -year OS probability forecast was $0.653 \pm 0.029$, the c-index of the LDH-CRS model was $0.674 \pm 0.029$, while the c-index of the mCRS model was $0.681 \pm 0.028$ (Fig. 4). These results suggest that adding LDH to the CRS scoring system demonstrated a better accuracy.

\section{Association of LDH levels and immune/inflammation-related indexes}

In an exploratory analysis, it is interesting to note that LDH levels varied with a set of immune/inflammatory factors (Fig. 5). Specifically, patients with elevated LDH had higher preoperative neutrophil counts $(P=0.031)$, higher $C$-reaction protein (CRP) levels $(P<.001)$, and lower lymphocyte counts $(P=0.022)$ than patients with normal LDH levels. Consequently, patients with elevated LDH also had a lower lymphocyte-to-monocyte ratio $(L M R ; P<.001)$ and lymphocyte-to-neutrophil ratio $(L N R ; P<$ .001). On the contrary, LDH levels were not associated with preoperative total white blood cell counts or monocyte counts.

\section{Discussion}

Resection of colorectal liver metastases is fraught with high rates of recurrence. It represents an area of intense investigation in desperate need of predictive biomarkers to aid in surgical decision-making. In the current study, we found that LDH was the strongest prognostic factor for OS both in the univariate and the multivariate analysis. Patients with elevated LDH had a nearly two-fold higher risk for mortality (mOS, 27.6 months vs. 68.8 months). The 5-year survival rate in the normal-LDH and the high-LDH groups was $53.7 \%$ and $22.5 \%$, respectively (Fig. 1). Although some scholars have investigated the utility of LDH as a serum biomarker in resectable and unresectable CRC, its usefulness has been limited by underpowered studies as well as its non-specificity (20-27). To the best of our knowledge, our study is the first to address the independent prognostic impact of preoperative LDH levels in curative-intent CRLM resection.

Increased LDH is closely linked to hypoxia and angiogenesis in aggressive tumor phenotypes showing accelerated growth kinetics (29-33). Metabolism of fast-growing cancer cells is shifted toward high glucose uptake and enhanced lactate production (34). In the TME, lactate promotes proinflammatory cytokines, such as TNF-a, IL-1, IL-6, prostaglandins, and nuclear factor-KB; enhances immune-suppressive cells, such as myeloid-derived suppressor cells (MDSCs) and dendritic cells (DCs); inhibits cytolytic cells, such as natural killer (NK) cells and cytotoxic T-lymphocytes (CTLs); recruit tumor-associated macrophages (TAMs) and promotes their conversion into immunosuppressive phenotype (35-37). Therefore, elevated LDH is a negative prognostic biomarker not only because its key role in cancer metabolism, but also because it modulates the complex interplay between the TME and the host immune system, impacting the proliferation, invasion, and migration potential of malignant tumors (38). 
Interestingly, exploratory analysis unexpectedly showed that LDH levels strongly correlated with systemic inflammation indexes, namely lymphocyte-to-monocyte ratio (LMR), lymphocyte-to-neutrophil ratio (LNR), and C-reaction protein. In contrast, this correlation was not observed for CEA levels (data not shown). It has been reported that systemic inflammation leads to lymphocytopenia and increased presence of TAMs, resulting in decreased cellular immunity (39-43). Meanwhile, growing evidence has shown that LDH could be a marker of diminished antitumor immunity, which inversely correlated with response to immune checkpoint blockade therapy (44). Moreover, the overexpression of hypoxia-regulating factors, such as HIF-1, Foxp3, and CCL-28, might contribute to an immunosuppressive microenvironment by recruiting myeloid-derived suppressor cells (MDSCs) and TAMs $(34,45)$. Thus, the mechanisms or pathways regulating LDH may intersect with hypoxia and antitumor immunity $(16,33,36)$. LDH may serve as an alternative indicator of systemic inflammation and immunosuppression. LDH is recently emerging as an anticancer target $(46,47)$. Herein, we postulate that the perioperative use of nonsteroidal anti-inflammatory drugs might decrease the recurrence risk of CRLM resection (48).

It was reported that LDH could be the product of tumor necrosis due to hypoxia, which is a sign of high tumor burden (16). In the present study, serum LDH levels did not show much relevance to most clinicopathological parameters (such as primary tumor sidedness, $\mathrm{T}$ and $\mathrm{N}$ stage, KRAS status, pathology and differentiation, and disease-free interval). Though elevated LDH were indeed associated with the maximum diameter of CRLM in our analysis, nevertheless, for patients with elevated LDH, 32.2\% (30/93) of them virtually had the maximum diameter of CRLM below median value $(2.5 \mathrm{~cm})$ (Table 2). It was also worth noting that LDH levels were not associated with the number of CRLM. Perhaps more importantly, subgroup analysis showed that the prognostic value of LDH was independent of the number and size of CRLM. LDH also demonstrated even strong prognostic value among patients with extra-hepatic metastases or with R1 surgical margin. Besides, LDH could distinguish the survival regardless of the Fong score (Fig. 2).

The above findings suggested that the prognostic attribute of LDH in the current study might go beyond a simple indicator of heavier tumor burden. High LDH levels might denote aggressive biology in a way that is independent of traditional molecular and clinicopathology features. LDH might be both a metabolic and an immune surveillance prognostic biomarker.

The prognostic scoring system proposed by Fong et al. has been widely used in clinical practice to stratify CRLM patients (28). This five-marker prognostic scoring system consists of baseline CEA levels ( $200 \mathrm{ng} / \mathrm{ml}$ as cut-off value), lymph node status of the primary tumor, number and size of hepatic metastases, and disease-free interval from the of the primary tumor. Though routine CEA test in CRC care is recommended globally, only $6.3 \%$ of patients in our dataset had CEA $>200 \mathrm{ng} / \mathrm{ml}$, while a higher proportion of patients (16\%) had elevated LDH. Consistent with a recent study (9), we found that CEA had insufficient statistical power to detect OS differences $(P=0.184)$. Notably, LDH could provide additional discriminatory ability on basis of CEA and CA19-9 levels. Specifically, among patients with CEA $>5 \mathrm{ng} / \mathrm{ml}$, median OS was distinguishable between patients with elevated and normal LDH levels ( 24.2 months vs. 60.6 months). For patients with CEA $<5 \mathrm{ng} / \mathrm{ml}$, elevated LDH still indicated worse OS (36.3 months vs. 
not reached). We observed even more significant trend for CA19-9 levels (Supplementary Fig. 2). Therefore, combing the Fong score with LDH, with a better prognostic discriminatory ability, outperformed the Fong score. Remarkably, both LDH-CRS and mCRS identified a relatively higher proportion of patients in the high-risk group (score $4-6)$ than CRS ( $13.2 \%$ vs. $12.0 \%$ vs. $8.5 \%)$. Thus, they could better define a portrait of the optimal candidate for CRLM resection with long-term survival, as well as a picture of patients in whom direct hepatectomy may be ill-advised and further neoadjuvant or adjuvant systemic therapy would be preferable.

We acknowledge that our analysis has some limitations due to its retrospective and observational nature. Some genetic parameters, including $R A S, B R A F$, microsatellite status, and post-relapse treatment, were not available in some datasets. It would be meaningful to combine LDH and specific mutations and molecular features of CRC in the future. Recurrence time was not thoroughly recorded in cohort 2. Estimating of RFS was not stringently carried out at protocol-specified intervals, though most physicians assessed the tumor status every 8-12 weeks. Less frequent assessment may bias in favor of a longer RFS time. Nevertheless, this factor is less likely to influence the primary OS outcome, which could genuinely reflect the clinical benefit of hepatectomy (49). Because the determination of the optimal cutoff value of LDH was beyond the scope of this study, we used the ULN to dichotomize this continuous variable, and the two participating centers adopted the same ULN of $250 \mathrm{U} / \mathrm{ml}$. Finally, the enrollment dates of the two cohorts differed. Prospectively defined resectability criteria for CRLM were not established in the study protocol, the therapeutic decisions were made by a multi-disciplinary team (MDT) in each medical center. However, since surgical interventions might outline a selection process per se, this would minimize the variations in patient selection between the two cohorts.

Anyhow, the above weaknesses had to be seen through the lens of the clear strengths. The advantage of our study resided in the large sample size, the division in two independent cohorts, the long-term followup, and the heterogeneous cohort of unselected, real-world patients. We also discovered that LDH might provide additional information on tumor metabolic and immune states. The accessibility and reproducibility of the noninvasive laboratory serum LDH test support its routine use in clinical practice. We expect future studies with prospective designs to validate our findings, and a more explicit understanding about the molecular mechanisms of LDH in governing tumor biology.

\section{Conclusion}

Our study implicates preoperative LDH level as a reliable and independent laboratory biomarker to predict the outcome of curative-intent surgery for CRLM. Integrating LDH into the established Fong scoring system can enhance the discrimination ability. Composite of LDH and Fong score is a potential stratification tool for CRLM resection. Prospective, international studies are needed to validate these results across diverse populations.

\section{List Of Abbreviations}


LDH, lactate dehydrogenase; ULN, upper limit of normal; CRLM, colorectal liver metastases; CRS, Clinical Risk Score; mCRS, modified Clinical Risk Score; HR, hazard ratio; $\mathrm{Cl}$, confidence interval; OS, overall survival; RFS, relapse-free survival.

\section{Declarations}

\section{Ethics approval and consent to participate}

The authors declare that ethical approval has been acquired from the Research Ethics Committee of Sun Yat-sen University Cancer Center and Sun Yat-sen University Sixth Affiliated Hospital for this retrospective analysis. All necessary informed consent required by applicable law has also been acquired from relevant patients. All methods were carried out in accordance with Helsinki guidelines. No further ethical approval was required.

\section{Consent for publication}

All authors have read and approved the final version to be published and signed the author disclosure form.

\section{Availability of data and materials}

The datasets used and/or analyzed during the current study are available from the corresponding author on reasonable request.

\section{Competing interests}

The authors declare that they have no conflicts of interest related to this research

\section{Funding}

This study was supported by National Natural Science Foundation of China (Grant No. 81802452, 82073302, 81602070, 81930065); Science and Technology Program of Guangzhou (2019B020227002, 201904020046, 201803040019, 201704020228); China Postdoctoral Science Foundation (2019M653216).

\section{Authors' contributions}

DSW: conceptualization, project administration, and supervision. ZZP: data curation and resources. YLW: conceptualization, data curation, and resources. LB: writing - original draft, and writing - review and editing. ZYL: writing - original draft. YXL: writing - original draft, methodology, software, and visualization. QC: data curation and resources. HZ: data curation and resources. YJ: data curation and resources. FW: data curation and resources. QM: methodology, software, and visualization.

\section{Acknowledgements}


We acknowledge the invaluable contributions of the patients who participated in this study and their families. We are thankful to Dr Qi Zhao from Bionformatic Core of our center for the helpful comments on the data analysis.

\section{References}

1. Torre L, Bray F, Siegel R, Ferlay J, Lortet-Tieulent J, Jemal AJCacjfc. Global cancer statistics, 2012. CA Cancer J Clin 2015;65(2):87-108.

2. Henley S, Ward E, Scott S, Ma J, Anderson R, Firth A, et al. Annual report to the nation on the status of cancer, part I: National cancer statistics. cancer. 2020;126(10):2225-49.

3. Feng RM, Zong YN, Cao SM, Xu RH. Current cancer situation in China: good or bad news from the 2018 Global Cancer Statistics? Cancer Commun (Lond). 2019;39(1):22.

4. Alberts SR. Update on the optimal management of patients with colorectal liver metastases. Crit Rev Oncol Hematol. 2012;84(1):59-70.

5. Mella J, Biffin A, Radcliffe AG, Stamatakis JD, Steele RJ. Population-based audit of colorectal cancer management in two UK health regions. Colorectal Cancer Working Group, Royal College of Surgeons of England Clinical Epidemiology and Audit Unit. Br J Surg. 1997;84(12):1731-6.

6. Ayez N, Burger JW, van der Pool AE, Eggermont AM, Grunhagen DJ, de Wilt JH, et al. Long-term results of the "liver first" approach in patients with locally advanced rectal cancer and synchronous liver metastases. Dis Colon Rectum. 2013;56(3):281-7.

7. Nordlinger B, Van Cutsem E, Gruenberger T, Glimelius B, Poston G, Rougier P, et al. Combination of surgery and chemotherapy and the role of targeted agents in the treatment of patients with colorectal liver metastases: recommendations from an expert panel. Ann Oncol. 2009;20(6):985-92.

8. Kulaylat AN, Schubart JR, Stokes AL, Bhayani NH, Wong J, Kimchi ET, et al. Overall survival by pattern of recurrence following curative intent surgery for colorectal liver metastasis. J Surg Oncol. 2014;110(8):1011-5.

9. Mavros M, Hyder O, Pulitano C, Aldrighetti L, Pawlik T. Survival of patients operated for colorectal liver metastases and concomitant extra-hepatic disease: external validation of a prognostic model. Journal of surgical oncology. 2013;107(5):481-5.

10. Tomlinson JS, Jarnagin WR, DeMatteo RP, Fong Y, Kornprat P, Gonen M, et al. Actual 10-year survival after resection of colorectal liver metastases defines cure. J Clin Oncol. 2007;25(29):4575-80.

11. Vigano L, Russolillo N, Ferrero A, Langella S, Sperti E, Capussotti L. Evolution of long-term outcome of liver resection for colorectal metastases: analysis of actual 5-year survival rates over two decades. Ann Surg Oncol. 2012;19(6):2035-44.

12. Ayez N, Burger J, van der Pool A, Eggermont A, Grunhagen D, de Wilt J, et al. Long-term results of the "liver first" approach in patients with locally advanced rectal cancer and synchronous liver metastases. Diseases of the colon and rectum. 2013;56(3):281-7. 
13. de Haas R, Wicherts D, Flores E, Azoulay D, Castaing D, Adam R. R1 resection by necessity for colorectal liver metastases: is it still a contraindication to surgery? Annals of surgery. 2008;248(4):626-37.

14. Hung HY, Chen JS, Chien Y, Tang R, Hsieh PS, Wen S, et al. Preoperative alkaline phosphatase elevation was associated with poor survival in colorectal cancer patients. Int $\mathrm{J}$ Colorectal Dis. 2017;32(12):1775-8.

15. Abdel-Rahman O. Prognostic Value of Baseline ALBI Score Among Patients With Colorectal Liver Metastases: A Pooled Analysis of Two Randomized Trials. Clin Colorectal Cancer. 2019;18(1):e61-e8.

16. Lukacova S, Sorensen BS, Alsner J, Overgaard J, Horsman MR. The impact of hypoxia on the activity of lactate dehydrogenase in two different pre-clinical tumour models. Acta Oncol. 2008;47(5):941-7.

17. Kolev $Y$, Uetake $H$, Takagi $Y$, Sugihara K. Lactate dehydrogenase-5 (LDH-5) expression in human gastric cancer: association with hypoxia-inducible factor (HIF-1alpha) pathway, angiogenic factors production and poor prognosis. Ann Surg Oncol. 2008;15(8):2336-44.

18. Gerlinger M, Wilson P, Powles T, Shamash J. Elevated LDH predicts poor outcome of recurrent germ cell tumours treated with dose dense chemotherapy. Eur J Cancer. 2010;46(16):2913-8.

19. Yue ZX, Xing TY, Gao C, Liu SG, Zhao W, Zhao Q, et al. Chromosome band 11q23 deletion predicts poor prognosis in bone marrow metastatic neuroblastoma patients without MYCN amplification. Cancer Commun (Lond). 2019;39(1):68.

20. Wu XZ, Ma F, Wang XL. Serological diagnostic factors for liver metastasis in patients with colorectal cancer. World J Gastroenterol. 2010;16(32):4084-8.

21. Connell LC, Boucher TM, Chou JF, Capanu M, Maldonado S, Kemeny NE. Relevance of CEA and LDH in relation to KRAS status in patients with unresectable colorectal liver metastases. J Surg Oncol. 2017;115(4):480-7.

22. Chibaudel B, Bonnetain F, Tournigand C, Bengrine-Lefevre L, Teixeira L, Artru P, et al. Simplified prognostic model in patients with oxaliplatin-based or irinotecan-based first-line chemotherapy for metastatic colorectal cancer: a GERCOR study. Oncologist. 2011;16(9):1228-38.

23. Lin J, Peng J, Zhao Y, Luo B, Zhao Y, Deng Y, et al. Early recurrence in patients undergoing curative resection of colorectal liver oligometastases: identification of its clinical characteristics, risk factors, and prognosis. J Cancer Res Clin Oncol. 2018;144(2):359-69.

24. Giessen C, Fischer von Weikersthal L, Laubender RP, Stintzing S, Modest DP, Schalhorn A, et al. Evaluation of prognostic factors in liver-limited metastatic colorectal cancer: a preplanned analysis of the FIRE-1 trial. Br J Cancer. 2013;109(6):1428-36.

25. Lisanti C, Basile D, Parnofiello A, Bertoli E, Andreotti V, Garattini S, et al. The SENECA study: Prognostic role of serum biomarkers in older patients with metastatic colorectal cancer. Journal of geriatric oncology. 2020.

26. Li G, Wang Z, Xu J, Wu H, Cai S, He Y. The prognostic value of lactate dehydrogenase levels in colorectal cancer: a meta-analysis. BMC Cancer. 2016;16(1). 
27. Caputo D, Caricato M, Vincenzi B, La Vaccara V, Mascianà G, Coppola R. Serum lactate dehydrogenase alone is not a helpful prognostic factor in resected colorectal cancer patients. Updates in surgery. 2014;66(3):211-5.

28. Fong Y, Fortner J, Sun RL, Brennan MF, Blumgart LH. Clinical score for predicting recurrence after hepatic resection for metastatic colorectal cancer: analysis of 1001 consecutive cases. Ann Surg. 1999;230(3):309-18; discussion 18-21.

29. Koukourakis MI, Giatromanolaki A, Simopoulos C, Polychronidis A, Sivridis E. Lactate dehydrogenase 5 (LDH5) relates to up-regulated hypoxia inducible factor pathway and metastasis in colorectal cancer. Clin Exp Metastasis. 2005;22(1):25-30.

30. Scartozzi M, Giampieri R, Maccaroni E, Del Prete M, Faloppi L, Bianconi M, et al. Pre-treatment lactate dehydrogenase levels as predictor of efficacy of first-line bevacizumab-based therapy in metastatic colorectal cancer patients. British journal of cancer. 2012;106(5):799-804.

31. Loupakis F, Intini R, Cremolini C, Orlandi A, Sartore-Bianchi A, Pietrantonio F, et al. A validated prognostic classifier for BRAF-mutated metastatic colorectal cancer: the 'BRAF BeCool' study. European journal of cancer (Oxford, England : 1990). 2019;118:121-30.

32. Kemeny N, Braun D. Prognostic factors in advanced colorectal carcinoma. Importance of lactic dehydrogenase level, performance status, and white blood cell count. The American journal of medicine. 1983;74(5):786-94.

33. Li F, Zhan L, Dong Q, Wang Q, Wang Y, Li X, et al. Tumor-Derived Exosome-Educated Hepatic Stellate Cells Regulate Lactate Metabolism of Hypoxic Colorectal Tumor Cells via the IL-6/STAT3 Pathway to Confer Drug Resistance. OncoTargets and therapy. 2020;13:7851-64.

34. Ding J, Karp JE, Emadi A. Elevated lactate dehydrogenase (LDH) can be a marker of immune suppression in cancer: Interplay between hematologic and solid neoplastic clones and their microenvironments. Cancer Biomark. 2017;19(4):353-63.

35. Basile D, Garattini SK, Corvaja C, Montico M, Cortiula F, Pelizzari G, et al. The MIMIC Study: Prognostic Role and Cutoff Definition of Monocyte-to-Lymphocyte Ratio and Lactate Dehydrogenase Levels in Metastatic Colorectal Cancer. Oncologist. 2020.

36. Van Wilpe S, Koornstra R, Den Brok M, De Groot JW, Blank C, De Vries J, et al. Lactate dehydrogenase: a marker of diminished antitumor immunity. Oncoimmunology. 2020;9(1):1731942.

37. Serganova I, Cohen IJ, Vemuri K, Shindo M, Maeda M, Mane M, et al. LDH-A regulates the tumor microenvironment via HIF-signaling and modulates the immune response. PLoS One. 2018;13(9):e0203965.

38. Graziano F, Ruzzo A, Giacomini E, Ricciardi T, Aprile G, Loupakis F, et al. Glycolysis gene expression analysis and selective metabolic advantage in the clinical progression of colorectal cancer. The pharmacogenomics journal. 2017;17(3):258-64.

39. Terzic J, Grivennikov S, Karin E, Karin M. Inflammation and colon cancer. Gastroenterology. 2010;138(6):2101-14 e5. 
40. Køstner A, Kersten C, Löwenmark T, Ydsten K, Peltonen R, Isoniemi H, et al. The prognostic role of systemic inflammation in patients undergoing resection of colorectal liver metastases: C-reactive protein (CRP) is a strong negative prognostic biomarker. Journal of surgical oncology. 2016;114(7):895-9.

41. Haruki K, Shiba H, Horiuchi T, Sakamoto T, Gocho T, Fujiwara Y, et al. Impact of the C-reactive protein to albumin ratio on long-term outcomes after hepatic resection for colorectal liver metastases. Am J Surg. 2017;214(4):752-6.

42. Okugawa Y, Toiyama Y, Yamamoto A, Shigemori T, Ide S, Kitajima T, et al. Lymphocyte-C-reactive Protein Ratio as Promising New Marker for Predicting Surgical and Oncological Outcomes in Colorectal Cancer. Annals of surgery. 2020;272(2):342-51.

43. Diakos $\mathrm{Cl}$, Charles KA, McMillan DC, Clarke SJ. Cancer-related inflammation and treatment effectiveness. Lancet Oncol. 2014;15(11):e493-503.

44. Koh YW, Kang HJ, Park C, Yoon DH, Kim S, Suh C, et al. The ratio of the absolute lymphocyte count to the absolute monocyte count is associated with prognosis in Hodgkin's lymphoma: correlation with tumor-associated macrophages. Oncologist. 2012;17(6):871-80.

45. Liu Q, Luo D, Cai S, Li Q, Li X. Circulating basophil count as a prognostic marker of tumor aggressiveness and survival outcomes in colorectal cancer. Clinical and translational medicine. 2020;9(1):6.

46. Gallo M, Sapio L, Spina A, Naviglio D, Calogero A, Naviglio S. Lactic dehydrogenase and cancer: an overview. Frontiers in bioscience (Landmark edition). 2015;20:1234-49.

47. Fiume L, Manerba M, Vettraino M, Di Stefano G. Inhibition of lactate dehydrogenase activity as an approach to cancer therapy. Future medicinal chemistry. 2014;6(4):429-45.

48. Schack A, Fransgaard T, Klein MF, Gögenur I. Perioperative Use of Nonsteroidal Anti-inflammatory Drugs Decreases the Risk of Recurrence of Cancer After Colorectal Resection: A Cohort Study Based on Prospective Data. Annals of Surgical Oncology. 2019;26(12):3826-37.

49. Pilz L, Manegold C, Schmid-Bindert G. Statistical considerations and endpoints for clinical lung cancer studies: Can progression free survival (PFS) substitute overall survival (OS) as a valid endpoint in clinical trials for advanced non-small-cell lung cancer? Translational lung cancer research. 2012;1(1):26-35.

\section{Tables}

Table 1. Patient clinicopathologic characteristics 


\begin{tabular}{|c|c|}
\hline \multirow{2}{*}{\multicolumn{2}{|c|}{$\begin{array}{c}\text { Variables } \\
\text { Patient characteristics }\end{array}$}} \\
\hline & Patient characteristics \\
\hline $\begin{array}{l}\text { Cohort } 1 \\
\text { Cohort } 2\end{array}$ & $434(74.8)$ \\
\hline Age: median (range) & $\begin{array}{l}146(25.2) \\
59(20-82)\end{array}$ \\
\hline Gender & \\
\hline Male & $385(66.4)$ \\
\hline Female & $195(33.6)$ \\
\hline \multicolumn{2}{|l|}{ Preoperative CEA } \\
\hline$>5 \mathrm{ng} / \mathrm{ml}$ & $349(60.1)$ \\
\hline $\begin{array}{l}\leq 5 \mathrm{ng} / \mathrm{ml} \\
\text { Missing }\end{array}$ & $26(35.5)$ \\
\hline \multicolumn{2}{|l|}{ Preoperative LDH } \\
\hline $\begin{array}{l}\text { Over ULN } \\
\text { Under UI N }\end{array}$ & $93(16.0)$ \\
\hline \multicolumn{2}{|l|}{ Survival outcome } \\
\hline $\begin{array}{l}\text { Median follow-up (month) } \\
\text { Median OS (month) }\end{array}$ & $\begin{array}{l}60.5 \text { (95\% CI, 57.5-63.5) } \\
59.5(95 \% \text { CI, 58.4-70.6) }\end{array}$ \\
\hline \multicolumn{2}{|l|}{ Primary tumor characteristics } \\
\hline \multicolumn{2}{|l|}{ Location $^{\text {a }}$} \\
\hline Right-sided & $139(24.0)$ \\
\hline Left-sided & $340(58.6)$ \\
\hline Missing & $101(17.4)$ \\
\hline \multicolumn{2}{|l|}{ Differentiation } \\
\hline Well/moderate & $459(79.1)$ \\
\hline Poor & $121(20.9)$ \\
\hline \multicolumn{2}{|l|}{ Pathology } \\
\hline Adenocarcinoma & $543(93.5)$ \\
\hline Signet-ring cell / & $38(6.5)$ \\
\hline \multicolumn{2}{|l|}{ mucinous carcinoma } \\
\hline \multicolumn{2}{|l|}{ Lymph node metastases } \\
\hline Absent & $197(34.0)$ \\
\hline Present & $305(52.6)$ \\
\hline Missing & 78 (13.4) \\
\hline \multicolumn{2}{|l|}{ CRLM characteristics } \\
\hline \multicolumn{2}{|l|}{ Maximum diameter of CRLM } \\
\hline$\leq 5 \mathrm{~cm}$ & $490(84.5)$ \\
\hline$>5 \mathrm{~cm}$ & 80 (13.8) \\
\hline Missing & $10(1.7)$ \\
\hline \multicolumn{2}{|l|}{ Number of CRLM } \\
\hline 1 & $262(45.1)$ \\
\hline$>1$ & 319 (54.9) \\
\hline \multicolumn{2}{|l|}{ Time of occurrence of CRLM } \\
\hline Synchronous & 440 (75.9) \\
\hline Metachronous & $140(24.1)$ \\
\hline \multicolumn{2}{|l|}{ KRAS } \\
\hline Wild type & $126(21.7)$ \\
\hline Mutated & $52(9.0)$ \\
\hline \multicolumn{2}{|l|}{ Extrahepatic metastases } \\
\hline Yes & \\
\hline No & $\begin{aligned} & 49(8.4) \\
& 531(91.6)\end{aligned}$ \\
\hline \multicolumn{2}{|l|}{ R0 resection } \\
\hline Yes & $504(86.9)$ \\
\hline No & $72(12.4)$ \\
\hline Missing & $4(0.7)$ \\
\hline Perioperative Chemotherap & \\
\hline
\end{tabular}


a Colorectal cancer arising in or proximal to the splenic flexure was defined as right-sided; arising distal to the splenic flexure was defined as left-sided.

Abbreviations: OS, overall survival; $\mathrm{LDH}$, lactate dehydrogenase; ULN, upper limit of normal; CRLM, colorectal liver metastases.

Table 2. Relation between patient characteristics and LDH levels in the pooled cohort 


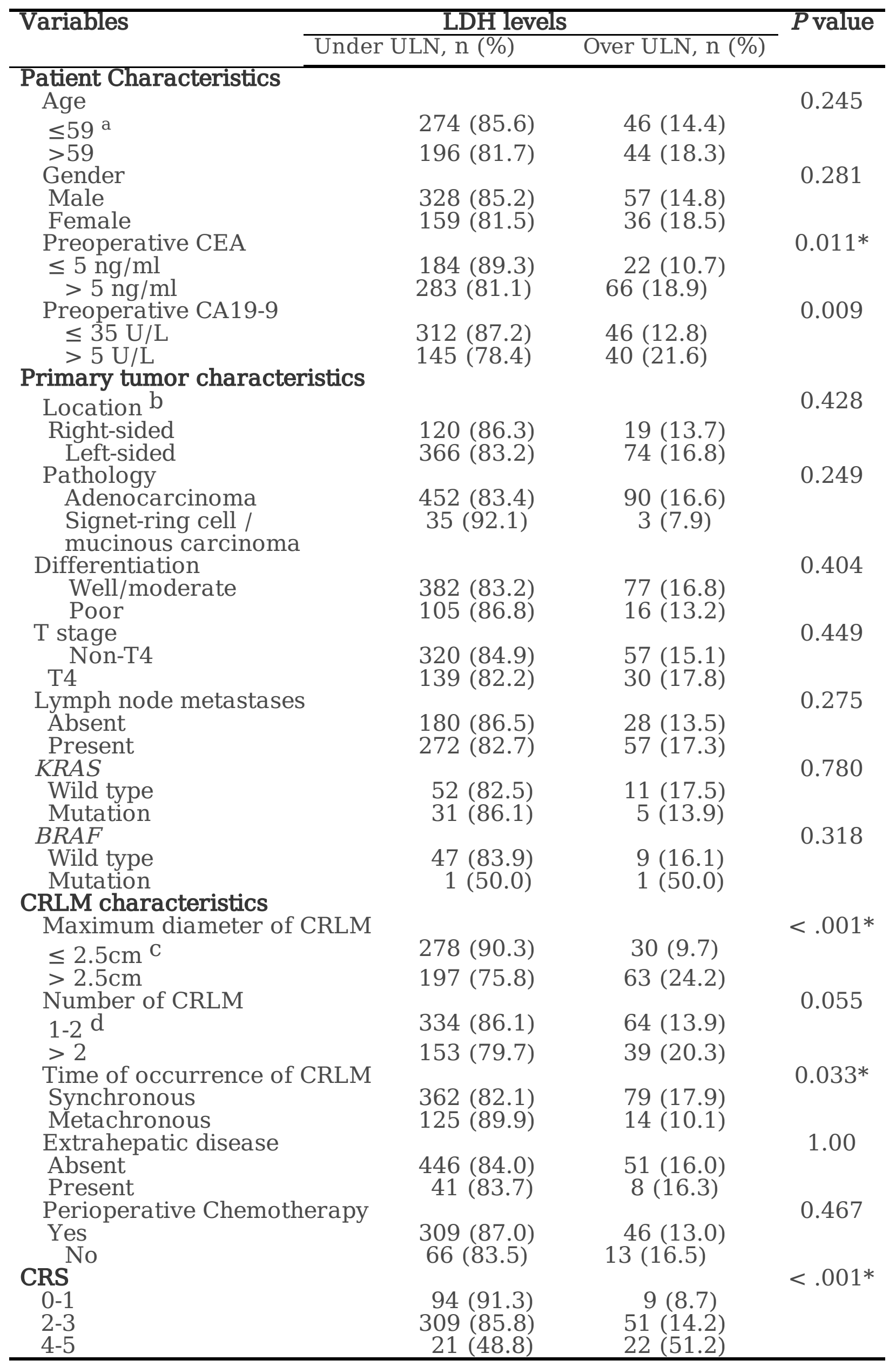


a Median age of patients in the pooled cohort was 59.

b Colorectal cancer arising in or proximal to the splenic flexure was defined as right-sided; arising distal to the splenic flexure was defined as left-sided.

C Median maximum diameter of CRLM in the pooled cohort was $2.5 \mathrm{~cm}$.

$\mathrm{d}$ Median number of CRLM in the pooled cohort was two.

Abbreviations: LDH, lactate dehydrogenase;

ULN, upper limit of normal; CRLM, colorectal liver metastases; CRS, Clinical Risk Score.

* indicates statistical significance.

Table 3. Univariate and multivariate analyses for predictors of overall survival in the pooled cohort 


\begin{tabular}{|c|c|c|c|c|}
\hline \multirow{2}{*}{ Variables } & \multicolumn{2}{|c|}{ Univariate analysis } & \multicolumn{2}{|c|}{ Multivariate analysis } \\
\hline & HR (95\% CI) & $P$ value & HR (95\% CI) & $P$ value \\
\hline Age & $1.02(1.006-$ & 0.002 & $1.03(1.01-$ & $<1 *$ \\
\hline Gender (male) & $1.19(0.92-1.54)$ & 0.192 & & \\
\hline $\begin{array}{l}\text { Primary tumor location } \\
\text { Right-sided vs. left-sided a }\end{array}$ & $1.17(0.88-1.54)$ & 0.277 & & \\
\hline $\begin{array}{l}\text { Poor differentiation } \\
\text { Signet-ring cell /mucinous }\end{array}$ & $\begin{array}{l}1.28(0.96-1.70) \\
1.09(0.69-1.73)\end{array}$ & $\begin{array}{l}0.088 \\
0.700\end{array}$ & $\begin{array}{l}0.99(0.70- \\
1.38)\end{array}$ & 1.38 \\
\hline $\begin{array}{l}\text { carcinoma } \\
\text { T4 stage }\end{array}$ & $1.30(1.00-1.68)$ & 0.050 & $1.26(0.94-$ & 0.126 \\
\hline $\begin{array}{l}\text { Primary tumor lymph node } \\
\text { metastasis }\end{array}$ & $1.78(1.36-2.33)$ & $<01$ & $\begin{array}{l}1.70(1.27- \\
227)\end{array}$ & $0<1 *$ \\
\hline $\begin{array}{l}\text { Preoperative CEA levels } \\
\text { Preoperative CA19-9 levels }\end{array}$ & $\begin{array}{l}1.67(1.24-2.26) \\
1.68(1.31-2.17)\end{array}$ & $\begin{array}{l}0.001 \\
< \\
.001\end{array}$ & $\begin{array}{c}1.23(0.91- \\
1.67)(1.09- \\
1.47 \quad\end{array}$ & $\begin{array}{l}0.184 \\
0.012^{*}\end{array}$ \\
\hline $\begin{array}{l}\text { Metachronous CRLM } \\
\text { Number of CRLM }\end{array}$ & $\begin{array}{l}0.82(0.63-1.07) \\
1.19(1.14-1.25)\end{array}$ & 0.151 & $1.13(1.07-$ & $1 *$ \\
\hline $\begin{array}{l}\text { Maximum diameter of CRLM } \\
\text { Extrahepatic metastases } \\
\text { Preoperative chemotherapy } \\
\text { R0 resection }\end{array}$ & $\begin{array}{l}1.09(1.04-1.13) \\
1.56(1.05-2.31) \\
1.51(1.19-1.92) \\
0.37(0.26-0.53)\end{array}$ & $\begin{array}{c}.001 \\
< \\
.001 \\
0.026 \\
0.001 \\
< \\
.001\end{array}$ & $\begin{array}{c}1.07(1.01- \\
1.13) \\
1.61(1.03- \\
2.54) \\
1.35(0.99- \\
1.86) \\
0.56(0.37- \\
0.84)\end{array}$ & $\begin{array}{l}0.027 * \\
0.039 * \\
0.061 \\
0.006^{*}\end{array}$ \\
\hline LDH levels (> ULN) & $2.51(1.88-3.36)$ & & $1.73(1.22-$ & $<$ \\
\hline Cohort 1 vs. cohort 2 & $0.89(0.65-1.21)$ & 0.886 & $\begin{array}{l}1.27(0.84- \\
1.93)\end{array}$ & 0.251 \\
\hline
\end{tabular}

a Colorectal cancer arising in or proximal to the splenic flexure were defined as right-sided, and those arising distal to the splenic flexure were defined as left-sided.

Abbreviations: HR, hazard ratio; CI, confidence interval; CRLM, colorectal liver metastases; ULN, upper limit of normal;

* indicates statistical significance.

\section{Figures}




\section{Figure 1}

\section{A Pooled cohort}

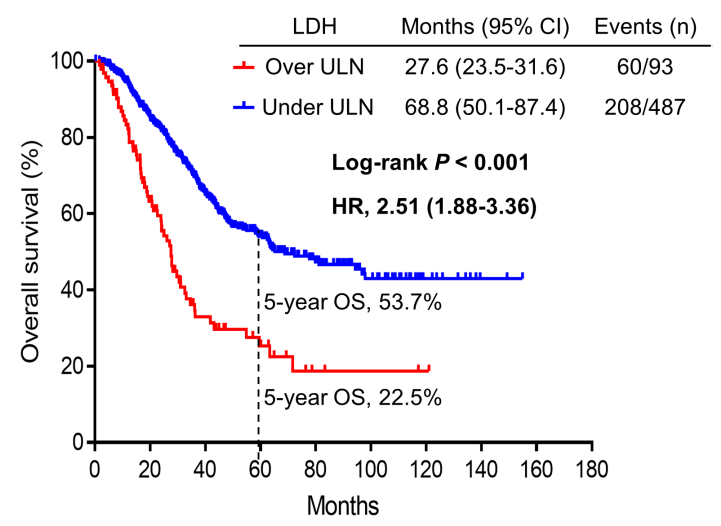

C

Cohort 2

\section{B}

Cohort 1

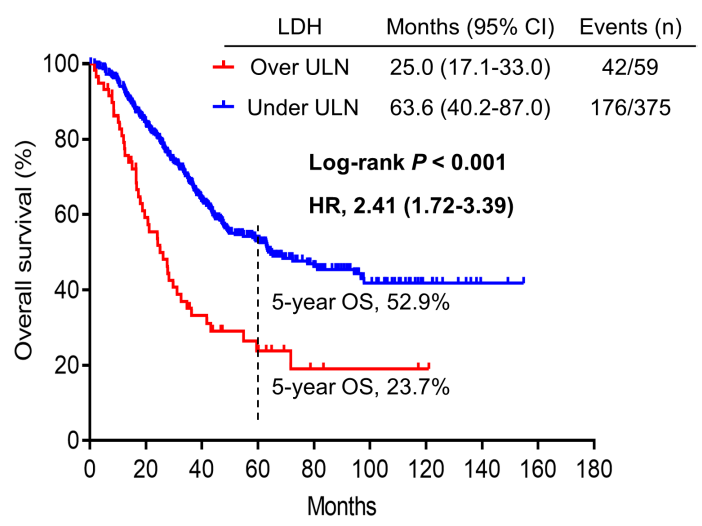

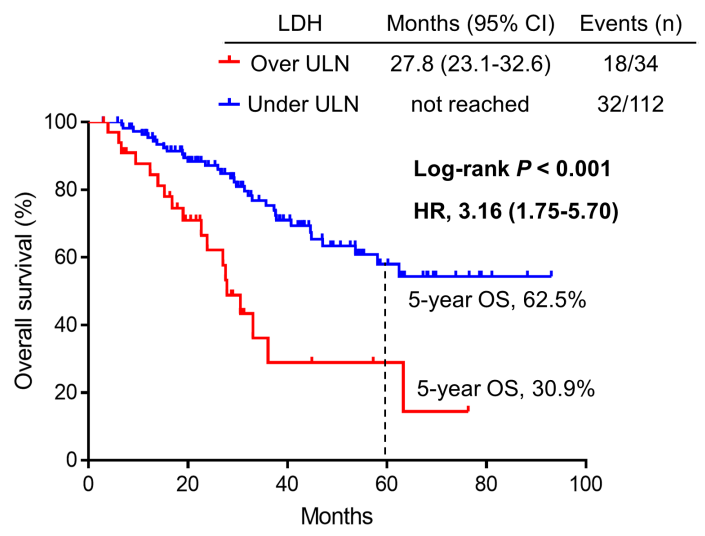

\section{Figure 1}

Overall survival according to preoperative serum LDH levels estimated by Kaplan-Meier curves. (A) Overall survival stratified by LDH levels in the pooled cohort. (B) Overall survival stratified by LDH levels in cohort 1. (C) Overall survival stratified by LDH levels in cohort 2. Abbreviations: LDH, lactate dehydrogenase; ULN, upper limit of normal; $\mathrm{Cl}$, confidence interval; $\mathrm{HR}$, hazard ratio. 
Figure 2

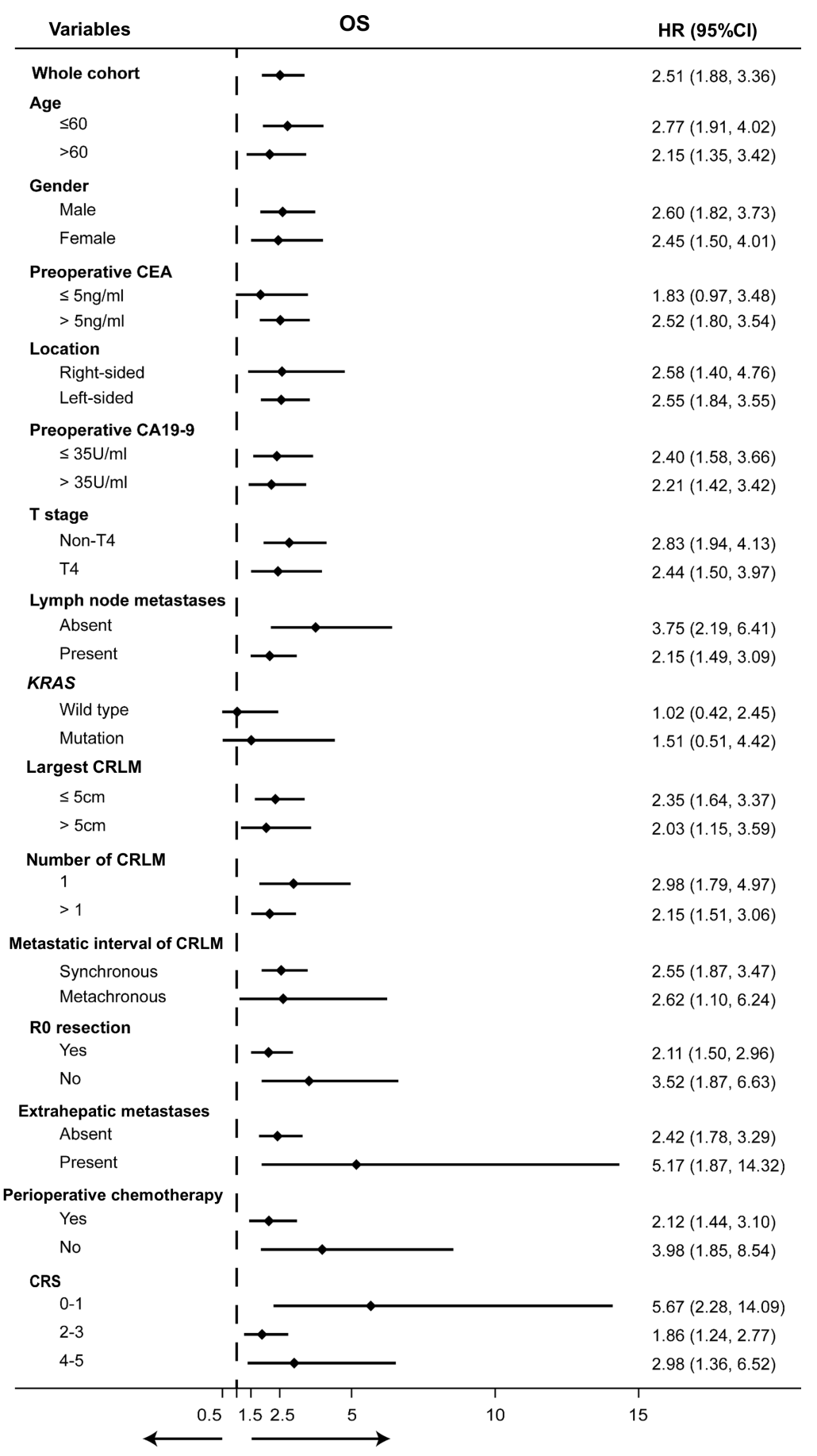

Favors higher LDH Favors lower LDH

\section{Figure 2}

Forest plots of hazard ratios (elevated LDH vs. normal LDH) for overall survival according to subgroups in the pooled cohort. Abbreviations: OS, overall survival; RFS, relapse-free survival; HR, hazard ratio; CRS, Clinical Risk Score. 


\section{Figure 3}

A

Pooled cohort

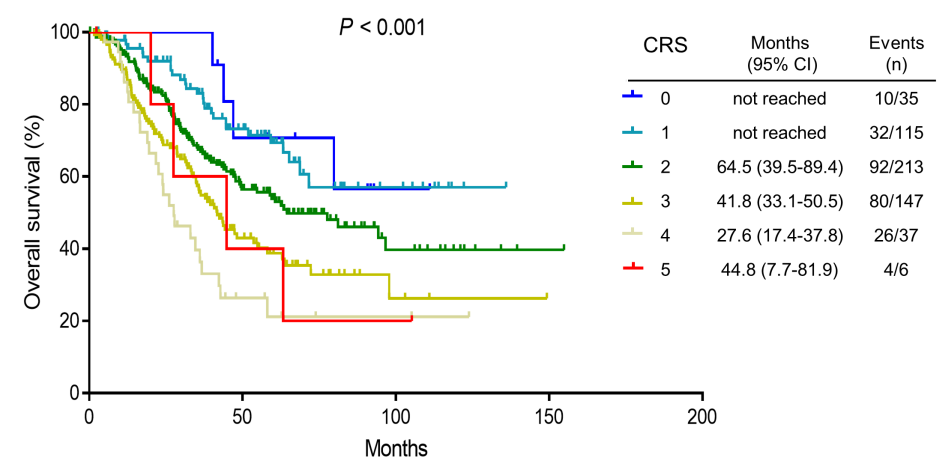

B

Pooled cohort

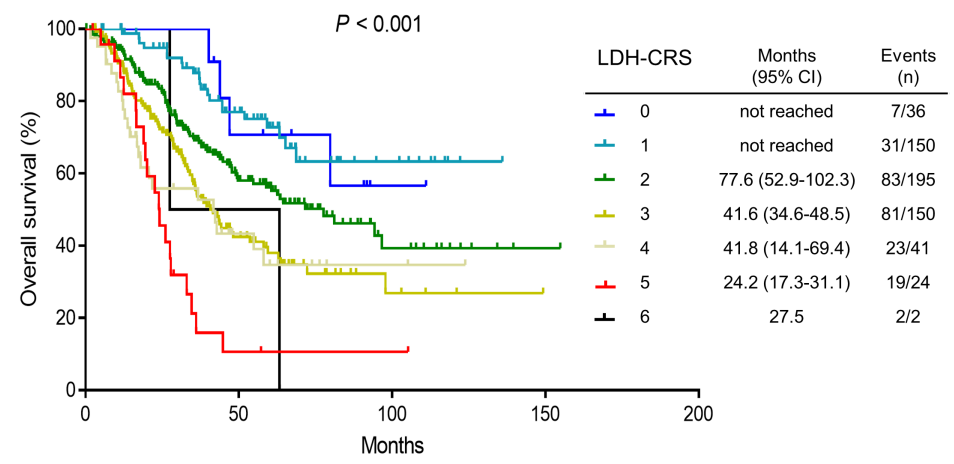

C

Pooled cohort

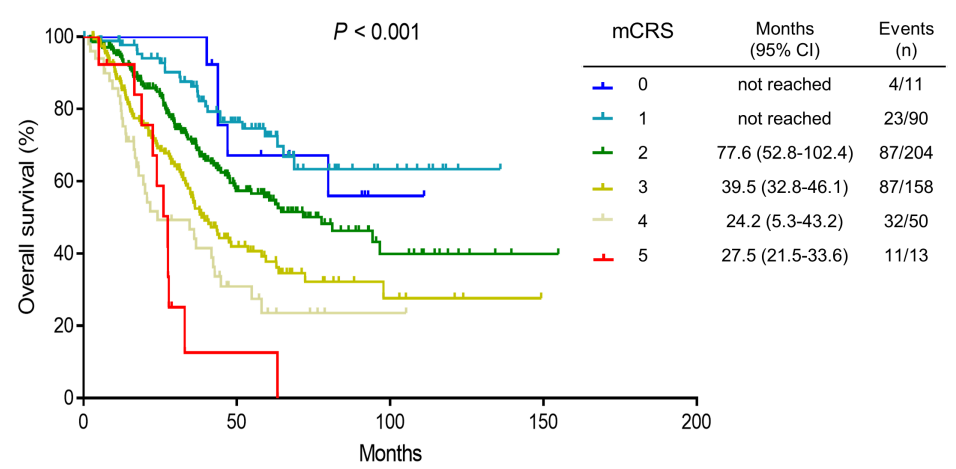

\section{Figure 3}

Overall survival stratified by different scoring systems in the pooled cohort. (A) Overall survival stratified by risk scores (0-5) as defined by CRS. (B) Overall survival stratified by risk scores (0-6) as defined by LDH-CRS. (C) Overall survival stratified by risk scores (0-5) as defined by modified CRS. Abbreviations: CRS, Clinical Risk Score; mCRS, modified Clinical Risk Score. 


\section{Figure 4}

\section{Pooled cohort}

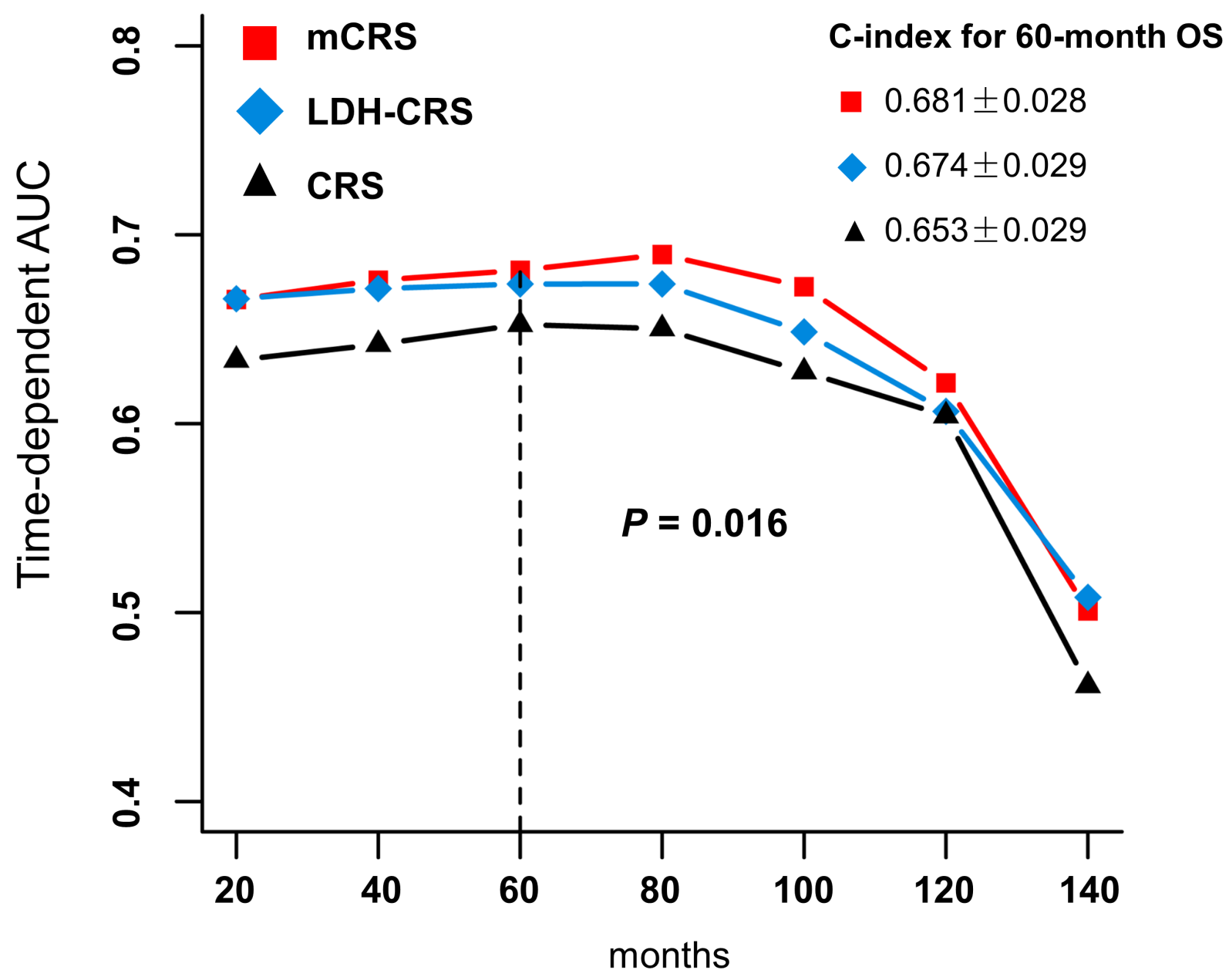

Figure 4

Receiver operating characteristic (ROC) analysis for the comparison of different scoring systems in prediction of overall survival in the pooled cohort. Abbreviations: CRS, Clinical Risk Score; mCRS, modified Clinical Risk Score; AUC, area under curve; C-index, concordance index; OS, overall survival. 


\section{Figure 5}

A

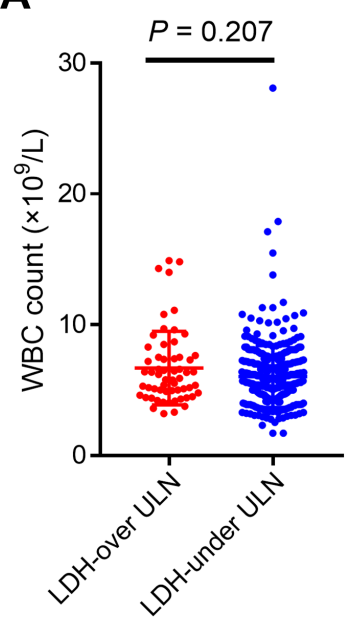

E

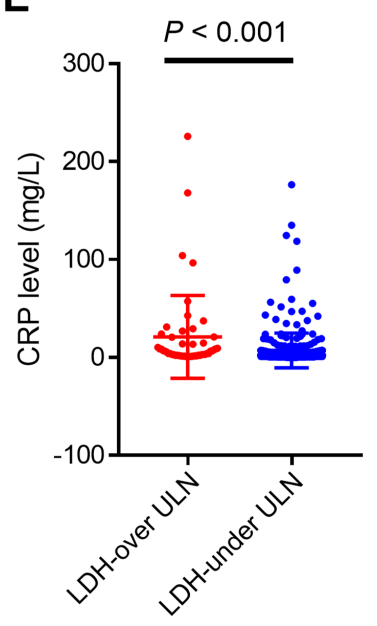

B

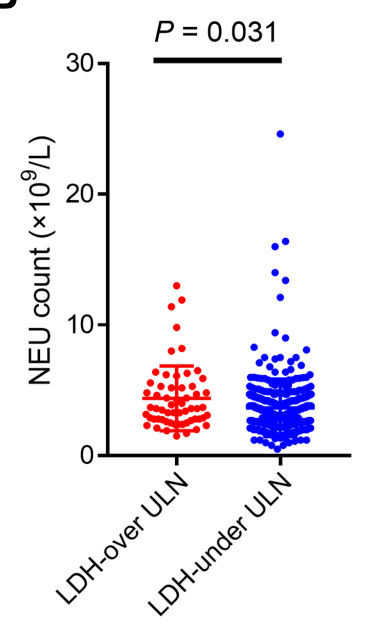

F

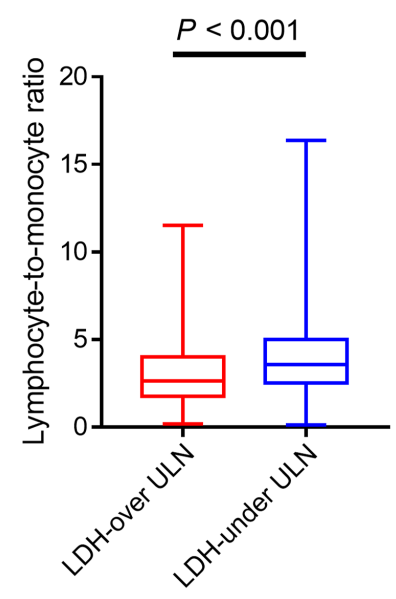

C

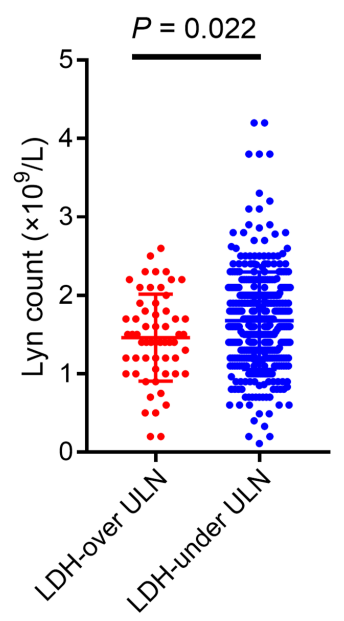

G

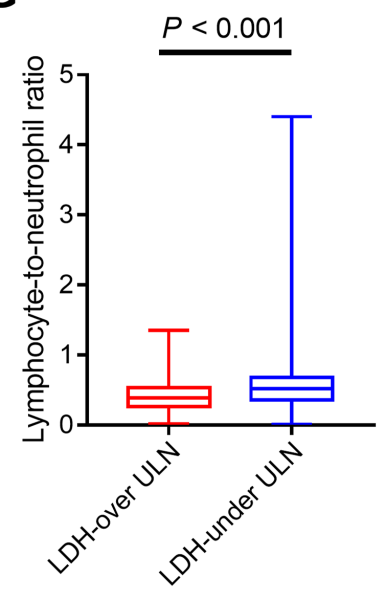

D

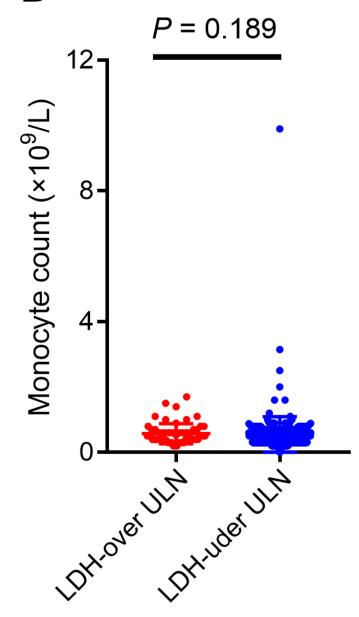

H

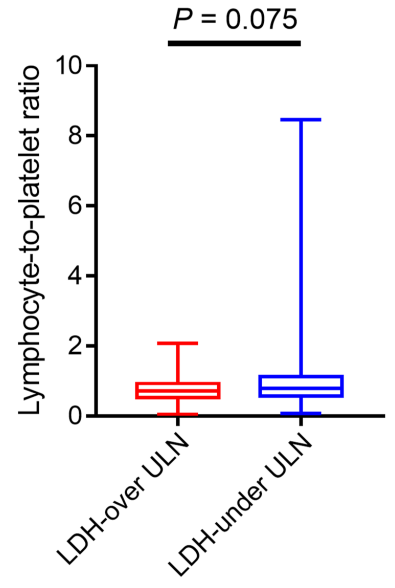

\section{Figure 5}

Associations between preoperative serum LDH levels and serum immune/inflammation-related factors. (A) LDH levels and WBC counts. (B) LDH levels and neutrophil counts. (C) LDH levels and lymphocyte counts. (D) LDH levels and monocyte counts. (E) LDH levels and C-reactive protein levels. (F) LDH levels and lymphocyte-to-monocyte ratios. (G) LDH levels and lymphocyte-to-neutrophil ratios. (H) LDH levels and lymphocyte-to-platelet ratios. Abbreviations: WBC, white blood cell; NEU, neutrophil; Lyn, lymphocyte; CRP, C-reactive protein.

\section{Supplementary Files}


This is a list of supplementary files associated with this preprint. Click to download.

- SupplementaryFigure1.tif

- SupplementaryFigure2.tif

- SupplementaryTable1.docx

- SupplementaryTable2.docx

- SupplementaryTable3.docx

- Supplementarytable4.docx

- SupplementaryTable5.docx 